\title{
A Comparative Analysis of the Value of Information in a Continuous Time Market Model with Partial Information: The Cases of Log-Utility and CRRA
}

\author{
Zhaojun Yang, ${ }^{1}$ Christian-Oliver Ewald, ${ }^{2}$ and Wen-Kai Wang ${ }^{2}$ \\ ${ }^{1}$ School of Finance and Statistics, Hunan University, Changsha 410079, China \\ ${ }^{2}$ School of Mathematics and Statistics, University of Sydney, Sydney NSW 2006, Australia \\ Correspondence should be addressed to Christian-Oliver Ewald, christian.ewald@sydney.edu.au
}

Received 16 March 2010; Accepted 12 May 2010

Academic Editor: Tak Kuen Siu

Copyright (c) 2011 Zhaojun Yang et al. This is an open access article distributed under the Creative Commons Attribution License, which permits unrestricted use, distribution, and reproduction in any medium, provided the original work is properly cited.

We study the question what value an agent in a generalized Black-Scholes model with partial information attributes to the complementary information. To do this, we study the utility maximization problems from terminal wealth for the two cases partial information and full information. We assume that the drift term of the risky asset is a dynamic process of general linear type and that the two levels of observation correspond to whether this drift term is observable or not. Applying methods from stochastic filtering theory we derive an analytical tractable formula for the value of information in the case of logarithmic utility. For the case of constant relative risk aversion (CRRA) we derive a semianalytical formula, which uses as an input the numerical solution of a system of ODEs. For both cases we present a comparative analysis.

\section{Introduction}

The economics of information and more precisely the way how information influences our strategic opportunities is a topic which is more and more discussed among economists, with applications in basically all behavioral sciences but particularly in finance. To an increase in strategic opportunities corresponds an increase in the level of maximal obtainable utility. This increase can be associated with a financial value and it is this value what is usually referred to as the value of information. This value depends in general on the whole model, assets, strategies, agents preferences, and so forth, and technically the same information can have different values for different agents and different underlying models. For more background on the general foundations of information economics we refer to Birchler and Büttler [1] 
and Hirshleifer and Riley [2]. In the specific context of stock markets the aspect of additional information and its value has been studied by various authors; see, for example, Amendinger et al. [3], Imkeller [4], Ewald [5], and Kohatsu-Higa and Sulem [6] as only to mention a view. While the first three focus on the case of initially enlarged filtration in the sense of Jacod [7], Kohatsu-Higa provides a rather general framework, which appears however to be to technical as to obtain analytical or numerical expressions for the value of information. All of the four models mentioned above treat the case of a representative agent, which either does or does not have an increased level of information, but there is no interaction between differently informed agents and no active exchange of information from one agent to another. The aspect of agent interaction and its strategic consequences in the presence of information asymmetry as well as the effect of increased information on general welfare has been studied by Ewald and Xiao [8]. In this article we resume the classical representative agent framework, but instead of initially enlarged information are study the case of partial information, in which the drift rate of the risky asset is assumed to be an unobservable process. The framework of partial information has been studied before for various levels of generality; see, for example, Pham and Quenez [9], Sass and Hausmann [10], Genotte [11], Dothan and Feldman [12], Detemple [13] and Xia [14]. Pham and Quenez study the case of a stochastic volatility model with partial information and derive formulas for the optimal strategy and the optimal wealth. The model discussed in our article could be treated along the same lines of arguments. However the formulas obtained by Pham and Quenez are not very explicit. By this we mean that from these formulas alone, it is difficult to say how model parameters effect the value of full information, which is one focus of our article. Sass and Haussmann are far more explicit and in fact provide a numerical and empirical analysis. Their discussion however is limited to the case where the unobserved process is a finite state Markov chain. Genotte studies a model which includes our model as a particular case, the setup is in fact far more general than ours, but the focus is on consumption rather than terminal wealth as in our article and no explicit formulas are derived. The articles of Dothan and Feldman as well as Detemple address different economic models, bond markets and interest rates in the first case, production economies in the second. Xia provides a quite general framework in which he also deals with an optimal consumption/terminal wealth problem under CRRA, but relies strongly on the numerical solution of the corresponding HJB equation via finite difference methods. None of these articles however really addresses the issue of the value of information. This extension could be easily done by adding the standard valuation method provided by information economics, however as indicated before, with the exception of Sass and Haussmann the obtained formulas are not explicit enough as to allow a numerical analysis and comparative statics. This is partly due to the high level of generality which was assumed in the underlying models. More explicit results at the expense of a loss in model generality are obtained by Yang and Ma [15]. Here the aspect of valuation of information is discussed but as an objective the maximization of consumption rather than expected utility from terminal wealth is chosen. Furthermore the model studied in our article is more general than by Yang and Ma [15] where a rather static setup was chosen. Focusing at first on the potentially easiest case of logarithmic utility but allowing a general linear dynamic for the unobserved drift term, we present a direct computation which leads to an explicit and analytically tractable expression for the value of full information in the case of a nonobservable drift term which is assumed to follow a linear stochastic differential equation. We then perform a comparative statics analysis and study how individual model parameters influence the value of the information. Such an analysis in the framework of a continuous time model has to the best of our knowledge not been done before. For the case of CRRA 
we derive a semianalytical formula for the value function under partial information and use this to determine the value of information under CRRA. More precisely, the Hamilton-JacobiBellman equation is reduced to a compact functional form, which as an input depends on the solution of a system of three ODEs. The numerical solution of this system of ODEs is far easier, than applying an implicit finite difference scheme in three dimensions, as done in Xia [14].

The remainder of the article is organized as follows. In Section 2, we introduce the investment model with partial information, while in Section 3, we compute the stochastic filtering estimate for the drift rate of the risky asset. We apply this result in Section 4 and give an explicit solution to the optimal investment problem with partial information under logarithmic utility. In Section 5, we provide a simple calculation formula for the information valuation while in Section 6 we study how individual parameters of the model influence the value of information. In Section 7, we repeat the analysis from the previous sections for the case of CRRA. Here we particularly focus on the effect of the risk-aversion parameter $\beta$ on the value of information. The main conclusions are summarized in Section 8 .

\section{The Partial Information Model}

We assume that there are two types of assets an investor can invest in: a risky asset and a risk-free asset. We think of the risky asset as a stock and the risk free asset as a savings deposit which pays a deterministic interest rate. The investment problem the agent faces is how to choose the appropriate ratio between risky asset and risk-free asset. For the case of complete information, where the agent is able to observe the noise generating process, this problem has been studied by many authors, most famously by Merton [16]. However, in principal it is unrealistic that the agent can observe the noise generating process, neither can he directly observe the parameters which constitute the model, for example the drift rate of the risky asset. What the agent does instead is observing the price process of both the risky asset and the risk-free asset. In general these processes carry less information than the noise generating process. The investment problem under partial information is the problem of how to invest optimally, when information is generated by the asset price processes only and not by the noise generating process. In the following we give a mathematical precise formulation at hand of an explicit example, which we continue to study in this article. Our agent faces a finite time horizon $[0, T]$ and an economic environment whose uncertainty is modeled by a complete probability space $(\Omega, \mathcal{F}, \mathbb{P})$. The prices of the two types of assets (risky and risk free) are denoted by $\left(B_{t}, S_{t}\right)$ and are governed by the following SDEs:

$$
\begin{aligned}
& d B_{t}=r_{t} B_{t} d t, \\
& d S_{t}=\mu_{t} S_{t} d t+\sigma_{t} S_{t} d W_{t}^{1},
\end{aligned}
$$

where the interest rate $r_{t}$ and the volatility $\sigma_{t}$ are deterministic processes, $W^{1}$ is a Brownian motion, and the appreciation rate $\mu_{t}$ is a stochastic process satisfying the following SDE:

$$
d \mu_{t}=\left(a_{1} \mu_{t}+a_{0}\right) d t+b_{1} d W_{t}^{1}+b_{2} d W_{t}^{2}, \quad 0 \leq t \leq T,
$$


with $a_{1}, a_{0}, b_{1}, b_{2}$ constants and $W^{2}$ a Brownian motion which is independent of $W^{1}$. The filtrations $\mathcal{F}=\left\{\mathcal{F}_{t}\right\}_{0 \leq t \leq T}$ and $\mathcal{G}=\left\{\mathcal{G}_{t}\right\}_{0 \leq t \leq T}$ with

$$
\mathcal{F}_{t}=\sigma\left(W_{s}^{1}, W_{s}^{2}: 0 \leq s \leq t\right), \quad \mathcal{G}_{t}=\sigma\left(S_{s}: 0 \leq s \leq t\right)
$$

represent the two different levels of information in our model. While the filtration $\mathcal{F}$ contains the information produced by the noise generating process the filtration $\mathcal{G}$ does only contain the information produced by the asset price processes. Note that since the bond price follows a deterministic process, it does not really contribute toward the information flow. We refer to $\mathcal{F}$ as full information and to $\mathcal{G}$ as partial information. Classically it is assumed that the investors have access to the full information flow $\left\{\mathcal{F}_{t}\right\}_{0 \leq t \leq T}$. This however means that the agent is able to observe the noise generating processes which in this case are the Brownian motions driving the stock price as well as the appreciation rate. This assumption is not very realistic. The case of partial information $\left\{\mathcal{G}_{t}\right\}_{0 \leq t \leq T}$ where the agent can only observe the asset prices is much more realistic. Let us now suppose that the investor has initial wealth $X_{0}$ and that she will invest according to a self-financing trading strategy. Suppose that at time $t \in[0, T]$, she invests a proportion $\pi_{t}$ of her wealth in the risky asset, and invests the rest in the risk-free asset. Then her wealth process $X=\left\{X_{t}\right\}_{0 \leq t \leq T}$ satisfies: for all $0 \leq t \leq T$,

$$
\begin{aligned}
d X_{t} & =\frac{\pi_{t} X_{t}}{S_{t}} d S_{t}+\frac{\left(1-\pi_{t}\right) X_{t}}{B_{t}} d B_{t} \\
& =\left(r_{t}+\pi_{t}\left(\mu_{t}-r_{t}\right)\right) X_{t} d t+\sigma_{t} \pi_{t} X_{t} d W_{t}^{1},
\end{aligned}
$$

where the process $\left\{\pi_{t}\right\}_{0 \leq t \leq T}$ must be $\mathcal{G}$-adapted. In summary, the optimal portfolio problem is described as follows:

$$
\max _{\left\{\pi_{t}\right\}_{0 \leq t \leq T}} \mathbb{E}\left[U\left(X_{T}\right)\right]
$$

subject to the constraints:

$$
\begin{aligned}
& d \mu_{t}=\left(a_{1} \mu_{t}+a_{0}\right) d t+b_{1} d W_{t}^{1}+b_{2} d W_{t}^{2}, \\
& d X_{t}=\left(r_{t}+\pi_{t}\left(\mu_{t}-r_{t}\right)\right) X_{t} d t+\sigma_{t} \pi_{t} X_{t} d W_{t}^{1},
\end{aligned}
$$

and $\left\{\pi_{t}\right\} \mathcal{G}_{\text {-adapted. }}\left\{S_{t}\right\}_{0 \leq t \leq T}$ and therefore also $\left\{X_{t}\right\}_{0 \leq t \leq T}$ can be observed, but $\left\{\mu_{t}\right\}_{0 \leq t \leq T}$ is the unobservable state process. Therefore, (2.5) and (2.6) are an optimization problem for a partially observable stochastic dynamic system. In the first part of this article the utility function $U$ is chosen to be logarithmic, that is, $U(x)=\log (x)$; later we consider the case of CRRA utility, that is, $U(x)=x^{\beta} / \beta$. 


\section{Filtering Estimation for the Average Appreciation Rate}

In this section, we apply the filtering technique to estimate the appreciation rate $\left\{\mu_{t}\right\}_{0 \leq t \leq T}$ given the information flow $\mathcal{G}$. Denote

$$
m_{t}=\mathbb{E}\left(\mu_{t} \mid \mathcal{G}_{t}\right), \quad \gamma_{t}=\mathbb{E}\left[\left(\mu_{t}-m_{t}\right)^{2} \mid \mathcal{G}_{t}\right]
$$

Note that $m_{0}=\mathbb{E}\left(\mu_{0}\right)$ and $\gamma_{0}=\operatorname{Var}\left(\mu_{0}\right)$. The following lemma follows from Theorem 11.1 by Liptser and Shiryayev [17].

Lemma 3.1. If the conditional distribution

$$
F_{\mathcal{G}_{0}}(x)=\mathbb{P}\left(\mu_{0} \leq x \mid \mathcal{G}_{0}\right)
$$

is normal with mean $m_{0}$ and variance $\gamma_{0}$, a.s., then the conditional distribution

$$
F_{\mathcal{G}_{t}}(x)=\mathbb{P}\left(\mu_{t} \leq x \mid \mathcal{G}_{t}\right)
$$

is normal a.s. with mean with $m_{t}$ and variance with $\gamma_{t}$.

As a normally distributed random variable is uniquely determined by its expectation and variance we conclude from this lemma that the knowledge of $m_{t}$ and $\gamma_{t}$ reflects all the information on $\mu_{t}$ the agent is able to obtain under the partial information $\mathcal{G}_{t}$.

Lemma 3.2. Let $\left\{\mu_{t}, S_{t}\right\}_{0 \leq t \leq T}$ be the stochastic processes with differentials given by (2.2) and (2.1). Suppose that $\mathbb{P}\left(\mu_{0} \leq x \mid \mathcal{G}_{0}\right)$ is Gaussian with mean $m_{0}$ and variance $\gamma_{0}$. Further, one assume that there exist two constants $0<c_{1}<c_{2}<\infty$ such that for all $t \in[0, T]$, one has $c_{1} \leq \sigma_{t} \leq c_{2}$. Then $m_{t}$ and $\gamma_{t}$ satisfy the following equations:

$$
\begin{aligned}
& d m_{t}=\left(a_{1} m_{t}+a_{0}\right) d t+\frac{b_{1} \sigma_{t}+\gamma_{t}}{\sigma_{t}^{2}}\left(\frac{d S_{t}}{S_{t}}-m_{t} d t\right), \\
& \dot{\gamma}_{t}=2 a_{1} \gamma_{t}+b_{1}^{2}+b_{2}^{2}-\left(\frac{b_{1} \sigma_{t}+\gamma_{t}}{\sigma_{t}}\right)^{2},
\end{aligned}
$$

where $\dot{\gamma}_{t}$ denotes the deterministic time-derivative of $\gamma_{t}$.

Proof. Applying Itô's formula to (2.1), we have

$$
d \log S_{t}=\left(\mu_{t}-\frac{1}{2} \sigma_{t}^{2}\right) d t+\sigma_{t} d W_{t}^{1}
$$

It is then easy to see that the conditions of Theorem 12.1 by Liptser and Shiryayev [17] hold for $(\mu, \log S)$. The conclusion of this lemma then follows from that theorem. 
In the case of the classic Black-Scholes model where $\sigma_{t}=\sigma$ is constant, it follows by using the technique of separation of variables, that $\gamma_{t}$ is explicitly given by the following expression:

$$
\gamma_{t}= \begin{cases}\gamma_{-}+2 \sqrt{\Lambda^{2}+b_{2}^{2} \sigma^{2}}\left(1-\frac{\gamma_{0}-\gamma_{+}}{\gamma_{0}-\gamma_{-}} \exp \left(-\frac{2 \sqrt{\Lambda^{2}+b_{2}^{2} \sigma^{2}}}{\sigma^{2}} t\right)\right)^{-1} & \text { if } \Lambda^{2}+b_{2}^{2} \neq 0 \\ \left(\frac{1}{\gamma_{0}}+\frac{t}{\sigma^{2}}\right)^{-1} & \text { if } \Lambda=b_{2}=0\end{cases}
$$

where

$$
\Lambda=a_{1} \sigma^{2}-b_{1} \sigma, \quad \gamma_{ \pm}=\Lambda \pm \sqrt{\Lambda^{2}+b_{2}^{2} \sigma^{2}}
$$

Remark 3.3. (1) The magnitude of $\gamma_{t}$ is a characterization of the accuracy of the estimate $m_{t}$ of $\mu_{t}$. If $\gamma_{t}$ converges to zero as $t \rightarrow \infty$, then $m_{t}$ is called a consistent estimate of $\mu_{t}$.

(2) It is obvious that $\gamma_{t} \rightarrow \gamma_{+}$as $t \rightarrow \infty$. Note that $\gamma_{+}=0$ if and only if $a_{1}=b_{1}=b_{2}=0$. Therefore, $m_{t}$ is consistent if and only if the appreciation rate is constant in time, that is, $\mu_{t} \equiv \mu_{0}$.

(3) The points $\gamma_{+}$and $\gamma_{-}$are stationary points of the dynamics of $\gamma_{t}$. As indicated in 2. $\gamma_{+}$is globally stable while $\gamma_{-}$is nonstable. In fact $\gamma_{-} \leq 0$ and as $\gamma_{0}>0$ it is easy to see that the trajectory of $\gamma_{t}$ is confined to the interval $\left[\min \left(\gamma_{0}, \gamma_{+}\right), \max \left(\gamma_{0}, \gamma_{+}\right)\right]$and furthermore that it is strictly monotonic.

Let us now define the innovation process by the following equation:

$$
d \bar{W}_{t}=\frac{1}{\sigma_{t}}\left(\frac{d S_{t}}{S_{t}}-m_{t} d t\right)
$$

It is well-known from the theory of filtering that $\bar{W}=\left\{\bar{W}_{t}\right\}_{0 \leq t \leq T}$ is a Brownian motion with respect to the stochastic basis $\left(\Omega, \mathcal{G}_{T}, \mathbb{P},\left\{\mathcal{G}_{t}\right\}_{0 \leq t \leq T}\right)$. Therefore, the self-financing condition (2.4) can be rewritten as

$$
d X_{t}=\left(r_{t} X_{t}+\left(m_{t}-r_{t}\right) \pi_{t} X_{t}\right) d t+\sigma_{t} \pi_{t} X_{t} d \bar{W}_{t}
$$

Similarly, combining (3.8) and (3.4), we obtain that

$$
d m_{t}=\left(a_{1} m_{t}+a_{0}\right) d t+\frac{b_{1} \sigma_{t}+\gamma_{t}}{\sigma_{t}} d \bar{W}_{t}
$$

The stochastic differential equations (3.9) and (3.10) describe the dynamics from the point of view of the partially informed agent. 


\section{Optimal Investment under Partial Information for Logarithmic Utility}

In this section we compute the optimal investment strategy under partial information for the case where the agents objective is maximization of expected logarithmic utility from terminal wealth. For this case we are able to obtain a reasonably tractable formula which allows us to study the effects of the model parameters on the value of the information. It follows from the Itô formula and (3.9) that

$$
\log X_{T}=\log X_{0}+\int_{0}^{T}\left(r_{t}+\left(m_{t}-r_{t}\right) \pi_{t}-\frac{1}{2} \sigma_{t}^{2} \pi_{t}^{2}\right) d t+\int_{0}^{T} \sigma_{t} \pi_{t} d \bar{W}_{t}
$$

Hence

$$
\mathbb{E}\left(\log X_{T}\right)=\log X_{0}+\int_{0}^{T} \mathbb{E}\left(r_{t}+\left(m_{t}-r_{t}\right) \pi_{t}-\frac{1}{2} \sigma_{t}^{2} \pi_{t}^{2}\right) d t
$$

whose maximum is attained at

$$
\pi_{t}=\frac{m_{t}-r_{t}}{\sigma_{t}^{2}}, \quad \forall t \in[0, T]
$$

The optimal value under partial information is then given by

$$
V_{P}=\log X_{0}+\int_{0}^{T}\left(r_{t}+\frac{\mathbb{E}\left(m_{t}-r_{t}\right)^{2}}{2 \sigma_{t}^{2}}\right) d t .
$$

From (3.10) we obtain that

$$
m_{t}=m_{0} e^{a_{1} t}+\frac{a_{0}}{a_{1}}\left(e^{a_{1} t}-1\right)+\int_{0}^{t} e^{a_{1}(t-s)}\left(b_{1}+\frac{\gamma_{s}}{\sigma_{s}}\right) d \bar{W}_{s}
$$

where in the case that $a_{1}=0$ the expression $\left(a_{0} / a_{1}\right)\left(e^{a_{1} t}-1\right)$ needs to be replaced by $a_{0} t$. We then obtain that

$$
\mathbb{E}\left(m_{t}-r_{t}\right)^{2}=\left(m_{0} e^{a_{1} t}+\frac{a_{0}}{a_{1}}\left(e^{a_{1} t}-1\right)-r_{t}\right)^{2}+\int_{0}^{t} e^{2 a_{1}(t-s)}\left(b_{1}+\frac{r_{s}}{\sigma_{s}}\right)^{2} d s
$$

Combining (4.4) and (4.6), we have

$$
\begin{aligned}
V_{P}= & \log X_{0}+\int_{0}^{T} r_{t} d t+\int_{0}^{T} \frac{\left(m_{0} e^{a_{1} t}+\left(a_{0} / a_{1}\right)\left(e^{a_{1} t}-1\right)-r_{t}\right)^{2}}{2 \sigma_{t}^{2}} d t \\
& +\int_{0}^{T} \int_{0}^{t} \frac{e^{2 a_{1}(t-s)}}{2 \sigma_{t}^{2}}\left(b_{1}+\frac{r_{s}}{\sigma_{s}}\right)^{2} d s d t .
\end{aligned}
$$


This expression only involves a deterministic integral, which can be computed using standard deterministic numerical integration methods. To the best of our knowledge no compact analytical expression for this integral exists in the general case. To summarize, we have the following theorem.

Theorem 4.1. At time $t \in[0, T]$, the optimal investment problem under partial information (2.5) with logarithmic utility subject to constraints (3.9) and (3.10) has the following solution:

$$
\pi_{t}=\frac{m_{t}-r_{t}}{\sigma_{t}^{2}}
$$

where $m_{t}$ is determined by (3.4). The optimal value function is given by (4.7).

It is important to identify (3.4) as the equation from which $m_{t}$ is obtained. Even though we derived a more explicit expression for $m_{t}$ in (4.6), the latter expression is not directly useful for the agent, as he does not observe the Brownian $\bar{W}$ but the stock $S$ instead.

Finally, we give an elementary example where the above quantities can be calculated in explicit form.

Example 4.2. Let us consider the special case that $\mu_{t} \equiv \mu_{0}$ is a normal random variable with mean $m_{0}$ and variance $\gamma_{0}$. Namely, $a_{1}=a_{0}=b_{1}=b_{2}=0$. Further, we take $r_{t}=r$ and $\sigma_{t}=\sigma$ to be constants. In this case

$$
\gamma_{t}=\left(\frac{1}{\gamma_{0}}+\frac{t}{\sigma^{2}}\right)^{-1}
$$

and hence

$$
V_{P}=\log X_{0}+r T+\frac{\left(m_{0}-r\right)^{2} T}{2 \sigma^{2}}-\frac{1}{2} \log \left(1+\frac{\gamma_{0} T}{\sigma^{2}}\right)+\frac{\gamma_{0} T}{2 \sigma^{2}}
$$

This example, which has also been studied by other authors; however is useless, when the question is "How do model parameters affect the value of information?", because in the setup of the example, there are to many coefficient restrictions and in fact the only remaining interesting parameters are $T$ and $\sigma$.

\section{Valuation of the Information under Logarithmic Utility}

In this section we determine the value of full information for an agent who has only partial information. As we said in the introduction, the value of a piece of information depends on how much information the individual agent already has. The value of the information is then obtained in the way, that the optimal expected utility the agent can achieve with his current level of information is subtracted from the optimal expected utility the agent could achieve with the increased level of information. The computed value of information is then in terms of additional utility. Alternatively a monetary value can be obtained by comparing certainty equivalences instead. However, here we choose utility as a scale. In order to fulfill this agenda in our particular model we need to compute the optimal expected logarithmic 
utility from terminal wealth under full information. This problem can in fact be solved along the same line of arguments as in the previous section for the case of partial information. There are however some minor subtleties and for completeness we include the argument. By Itô's formula, we have

$$
\log X_{T}=\log X_{0}+\int_{0}^{T}\left(r_{t}+\left(\mu_{t}-r_{t}\right) \pi_{t}-\frac{1}{2} \sigma_{t}^{2} \pi_{t}^{2}\right) d t+\int_{0}^{T} \sigma_{t} \pi_{t} d W_{t}^{1}
$$

Hence

$$
\mathbb{E} \log X_{T}=\log X_{0}+\int_{0}^{T} \mathbb{E}\left(r_{t}+\left(\mu_{t}-r_{t}\right) \pi_{t}-\frac{1}{2} \sigma_{t}^{2} \pi_{t}^{2}\right) d t
$$

whose maximum is attained at

$$
\pi_{t}^{*}=\frac{\mu_{t}-r_{t}}{\sigma_{t}^{2}}
$$

The value function is then given by

$$
V_{F}=\log X_{0}+\int_{0}^{T}\left(r_{t}+\frac{\mathbb{E}\left(\mu_{t}-r_{t}\right)^{2}}{2 \sigma_{t}^{2}}\right) d t
$$

By (2.6), we obtain that

$$
\mu_{t}=\mu_{0} e^{a_{1} t}+\frac{a_{0}}{a_{1}}\left(e^{a_{1} t}-1\right)+\int_{0}^{t} e^{a_{1}(t-s)}\left(b_{1} d W_{s}^{1}+b_{2} d W_{s}^{2}\right)
$$

It then follows from $\mathbb{E}\left(\mu_{0}\right)=m_{0}$ that

$$
\mathbb{E}\left(\mu_{t}-r_{t}\right)^{2}=e^{2 a_{1} t} \gamma_{0}+\left(m_{0} e^{a_{1} t}+\frac{a_{0}}{a_{1}}\left(e^{a_{1} t}-1\right)-r_{t}\right)^{2}+\frac{b_{1}^{2}+b_{2}^{2}}{2 a}\left(e^{2 a_{1} t}-1\right) .
$$

Combining (5.4) and (5.6), we see that

$$
\pi_{t}^{*}=\frac{\mu_{t}-r_{t}}{\sigma_{t}^{2}}
$$

and the optimal obtainable expected utility is given by

$$
V_{F}=\log X_{0}+\int_{0}^{T} r_{t} d t+\int_{0}^{T} \frac{1}{2 \sigma_{t}^{2}}\left(e^{2 a_{1} t} \gamma_{0}+\left(m_{0} e^{a t}+\frac{a_{0}}{a_{1}}\left(\mathrm{e}^{a_{1} t}-1\right)-r_{t}\right)^{2}+\frac{b_{1}^{2}+b_{2}^{2}}{2 a}\left(e^{2 a_{1} t}-1\right)\right) d t
$$


with $\left(a_{0} / a_{1}\right)\left(e^{a_{1} t}-1\right)$ replaced by $a_{0} t$ in the case that $a_{1}=0$. Following the program described above we then obtain the following theorem which associates a numerical value to full information relative to partial information.

Theorem 5.1. The value of full information relative to partial information in the model described in Section 2 is given by

$$
\mho=\int_{0}^{T} \frac{1}{2 \sigma_{t}^{2}}\left(e^{2 a_{1} t} \gamma_{0}+\frac{b_{1}^{2}+b_{2}^{2}}{2 a_{1}}\left(e^{2 a_{1} t}-1\right)-\int_{0}^{t} e^{2 a_{1}(t-s)}\left(b_{1}-\frac{\gamma_{s}}{\sigma_{s}}\right)^{2} d s\right) d t
$$

Proof. As indicated above we simply have to compute the value $V_{F}-V_{P}$. The valuation formula is then obtained by substitution of (5.8) and (4.7).

Perhaps the most striking thing about Theorem 5.1. is that the parameter $a_{0}$ from the non observable drift term does not have any influence on the value of information. We will include a comparative static analysis on how the individual model parameters influence this value in the next section. For the moment let us come back to Example 4.2. In this case we obtain the following.

Example 5.2. For the same choice of coefficients as in Example 4.2. we obtain that the value of full information relative to partial information is given by

$$
\mho=\frac{1}{2} \log \left(1+\frac{\gamma_{0} T}{\sigma^{2}}\right)
$$

\section{Comparative Statics and Numerical Analysis: Log-Utility}

In this section we restrict our analysis to the case where $\sigma_{t} \equiv \sigma$ is constant and investigate the effect changes of the model parameters $a_{1}, a_{0}, b_{1}, b_{2}$ and $\sigma$ have on the value of full information relative to partial information. For this case we have an explicit representation of the dynamic of the conditional variance $\gamma_{t}=\operatorname{var}\left(\mu_{t} \mid \mathcal{G}_{t}\right)$. We have already indicated in the previous section that the constant term $a_{0}$ in the unobservable drift term does not have any effect on the value of the information. The reason for this is that this parameter is not multiplied with a variable that produces uncertainty, that is, $\mu_{t}$, respectively, $m_{t}$ in the case of $a_{1}, d W^{1}$ in the case of $b_{1}$ and $\sigma_{1}$ and $d W^{2}$ in the case of $b_{2}$. The second observation which is easy to make is that an increase in the absolute value $\left|b_{2}\right|$ of the parameter $b_{2}$ always leads to an increase in the value of full information. Mathematically this can be seen as follows. Assume first that $a_{1} \neq 0$. It then follows directly from the valuation formula in Theorem 5.1. that

$$
\frac{\partial U}{\partial b_{2}}=\frac{b_{2}}{a_{1}} \int_{0}^{T}\left(e^{2 a_{1} t}-1\right) d t
$$


We have $\left(1 / a_{1}\right)\left(e^{2 a_{1} t}-1\right)>0$ for all choices of $a_{1}$ and positive $t$ and therefore our assertion holds. In the case that $a_{1}=0$ we obtain by taking the limit for $a_{1} \rightarrow 0$ in (6.1) that

$$
\frac{\partial \mho}{\partial b_{2}}=b_{2} \int_{0}^{T} 2 t d t=b_{2} T^{2}
$$

and we see that our assertion holds in this case as well. The economical interpretation of this result is more or less clear. The parameter $b_{2}$ increases the uncertainty in the unobserved process $\mu_{t}$ and decreases the covariation between asset and unobserved noise $b_{2} d W^{2}$. Information about this process therefore becomes more valuable the higher $\left|b_{2}\right|$ is. The effect of $b_{1}$ on the value of full information is more diversified and difficult to identify. Intuitively we can find two effects here. The first one is that an increase in $b_{1}$ (as does an increase in $b_{2}$ ) increase the uncertainty in the unobserved process $\mu_{t}$. However, an increase in $b_{1}$ also leads to an increase rather than a decrease of the covariation between $\mu_{t}$ and the risky asset $S_{t}$. While the first effect gives an inertia to an increase of the value of full information, the second effect gives an inertia to decrease its value. Formally by taking derivatives we see that

$$
\begin{aligned}
\frac{\partial \mathcal{U}}{\partial b_{1}}= & 2 b_{1} \int_{0}^{T} \int_{0}^{t} e^{2 a_{1}(t-s)}\left(1-\left(b_{1}-\frac{\gamma_{s}}{\sigma}\right)^{2}\right) d s d t \\
& +2 b_{1}^{2} \int_{0}^{T} \int_{0}^{t} e^{2 a_{1}(t-s)}\left(b_{1}-\frac{\gamma_{s}}{\sigma}\right) d s d t .
\end{aligned}
$$

Since $\gamma_{s} \in\left[0, \gamma_{+}\right]$for all $t \in[0, T]$, we see that this is positive, whenever

$$
\frac{\gamma_{+}}{\sigma}<b_{1}<1
$$

Economically it is reasonable to assume that the volatilities $b_{1}$ and $b_{2}$ of the drift term are less than 1. Remembering that $\gamma_{+}=\lim _{t \rightarrow \infty} \gamma_{t}$ represents the ultimate level of informational uncertainty and $\sigma$ the asset volatility, which in a way represents unresolvable model uncertainty, (6.4) can be interpreted as saying that informational uncertainty on the drift rate has to be comparably small relative to overall model uncertainty in order for $b_{1}$ to have a positive affect on the value of full information. Let us now consider the individual effect of $\sigma$. We have

$$
\frac{\partial \mho}{\partial \sigma}=-\frac{\gamma_{0}}{2 a_{1} \sigma_{0}^{3}}\left(e^{2 a_{1} T}-1\right)+2\left(b_{1}^{2}+b_{2}^{2}\right) \int_{0}^{T} \int_{0}^{t} e^{2 a_{1}(t-s)}\left(b_{1}-\frac{\gamma_{s}}{\sigma}\right) \frac{\gamma_{s}}{\sigma^{2}} d s d t
$$

where the first summand needs to be replaced by $-\gamma_{0} T / \sigma_{0}^{3}$ in the case that $a_{1}=0$. We are not able to provide a compact and conclusive analytical answer to the question whether this expression is positive or not at least for the general case. Numerically however it is easy to evaluate the integral and compute this expression. Obviously as can be seen from expression (6.5) high values of $b_{1}$ and $b_{2}$ increase the chance that $\partial \mho / \partial \sigma$ is positive. In the case that $b_{1} \leq \min \left(\gamma_{0}, \gamma_{+}\right) / \sigma$ we see that both summands in (6.5) are negative and hence in this case $\partial \mho / \partial \sigma<0$. Similarly it can be seen that if condition (6.4) holds we have $\partial \mho / \partial T>0$ which 
means that the value of information increases with the length of the time horizon the agent is facing. For the special case studied in Examples 4.2 and 5.2 things are more straightforward, however as indicated before, interesting effects are lost. It is easy to see from formula (5.10) that in this special case the larger the variability $\gamma_{0}$ of the drift rate $\mu_{0}$ or the time $T$, the larger the value of the information while the larger the asset volatility $\sigma$ of the risky asset, the smaller the value of the information. Returning back to the general case, Figures 1-6 in Appendices A and B illustrate our theoretical findings above. To produce these figures and in order to evaluate the double integral in Theorem 5.1 we used the following approach based on the classical Simpson rule. Consider a double integral

$$
\int_{a}^{b} \int_{c(x)}^{d(x)} f(x, y) d y d x
$$

Let the step size for $\mathrm{x}$ be $h=(b-a) / 2$ and let the step size for $y$ be $k(x)=(d(x)-c(x)) / 2$. Adapting the classical Simpson method iteratively, we obtain

$$
\begin{aligned}
& \int_{a}^{b} \int_{c(x)}^{d(x)} f(x, y) d y d x \\
& \approx \int_{a}^{b} \frac{k(x)}{3}[f(x, c(x))+4 f(x, c(x)+k(x))+f(x, d(x))] d x \\
& \approx \frac{h}{3}\left\{\frac{k(a)}{3}[f(a, c(a))+4 f(a, c(a)+k(a))+f(a, d(a))]\right. \\
& \quad+\frac{4 k(a+h)}{3}[f(a+h, c(a+h))+4 f(a+h, c(a+h)+k(a+h))+f(a+h, d(a+h))] \\
& \left.\quad+\frac{k(b)}{3}[f(b, c(b))+4 f(b, c(b)+k(b))+f(b, d(b))]\right\} .
\end{aligned}
$$

In Theorem 5.1, we need to evaluate

$$
\mho=\int_{0}^{T} \frac{1}{2 \sigma_{t}^{2}}\left[e^{2 a_{1} t} \gamma_{0}+\frac{b_{1}^{2}+b_{2}^{2}}{2 a_{1}}\left(e^{2 a_{1} t}-1\right)-\int_{0}^{t} e^{2 a_{1}(t-s)}\left(b_{1}-\frac{\gamma_{s}}{\sigma}\right)^{2} d s\right] d t .
$$

We divide $\mho$ into two parts. The first one is a single integral and the other is a double integral. Let $n$ be the number of discretized steps in time and $\Delta t=T / n$. Then

$$
t_{i}=t_{i-1}+\Delta t, \quad \text { for } i=1, \ldots, n, t_{0}=0
$$

We evaluate the first part by applying the standard Simpson method. Let $h=\Delta t / 2, \sigma_{t}=\sigma$ be a constant, and

$$
f(t)=\frac{1}{2 \sigma^{2}}\left[e^{2 a_{1} t} \gamma_{0}+\frac{b_{1}^{2}+b_{2}^{2}}{2 a_{1}}\left(e^{2 a_{1} t}-1\right)\right] .
$$


We obtain

$$
\sum_{i=0}^{n-1} \int_{t_{i}}^{t_{i+1}} \frac{1}{2 \sigma^{2}}\left[e^{2 a_{1} t} \gamma_{0}+\frac{b_{1}^{2}+b_{2}^{2}}{2 a_{1}}\left(e^{2 a_{1} t}-1\right)\right] d t \approx \sum_{i=0}^{n-1} \frac{h}{3}\left[f\left(t_{i}\right)+4 f\left(t_{i}+h\right)+f\left(t_{i+1}\right)\right]
$$

To evaluate the double integral, we let

$$
g(s, t)=\frac{e^{2 a_{1}(t-s)}\left(b_{1}-\gamma_{s} / \sigma\right)^{2}}{2 \sigma^{2}}
$$

By applying our adapted Simpson method (6.7) and choosing

$$
k(t)=\frac{t-0}{2}=\frac{t}{2}
$$

we obtain the following approximate integration formula:

$$
\begin{aligned}
& \sum_{i=0}^{n-1} \int_{t_{i}}^{t_{i+1}} \int_{0}^{t} g(s, t) d s d t \\
& \approx \sum_{i=0}^{n-1} \int_{t_{i}}^{t_{i+1}} \frac{t}{6}\left[g(0, t)+4 g\left(\frac{t}{2}, t\right)+g(t, t)\right] d t \\
& \approx \sum_{i=0}^{n-1} \frac{h}{3}\left\{\frac{t_{i}}{6}\left[g\left(0, t_{i}\right)+4 g\left(\frac{t_{i}}{2}, t_{i}\right)+g\left(t_{i}, t_{i}\right)\right]\right. \\
& \quad+\frac{2\left(t_{i}+h\right)}{3}\left[g\left(0, t_{i}+h\right)+4 g\left(\frac{t_{i}+h}{2}, t_{i}+h\right)+g\left(t_{i}+h, t_{i}+h\right)\right] \\
& \left.\quad+\frac{t_{i+1}}{6}\left[g\left(0, t_{i+1}\right)+4 g\left(\frac{t_{i+1}}{2}, t_{i+1}\right)+g\left(t_{i+1}, t_{i+1}\right)\right]\right\} .
\end{aligned}
$$

\section{The Value of Full Information under CRRA}

In this section, we consider the case where the agent's utility function in (2.5) is given by $U(x)=x^{\beta} / \beta$. This utility function is known as CRRA-utility. The parameter $\beta$ measures how risk averse the agent is. The case $\beta=1$ corresponds to risk neutrality, $\beta<1$ represents a risk averse agent while $\beta>1$ would represent a risk-seeking agent. The case $\beta=0$ formally corresponds to the case of logarithmic utility that we discussed in the previous sections.

It follows from classical stochastic optimal control theory that the value function for the problem of maximizing (2.5) subject to constraints (3.9), (3.10), that is, under partial information,

$$
J^{p}(t, m, x)=\max _{\left\{\pi_{u} \in \mathcal{G}_{u}\right\}_{t \leq u \leq T}} \mathbb{E}\left[U\left(X_{T}\right) \mid, m_{t}=m, X_{t}=x\right]
$$


satisfies the following Hamilton-Jacobi-Bellman equation:

$$
\begin{aligned}
0=\max _{\pi}( & J_{t}^{p}+a m J_{m}^{p}+\left(r_{t}+\left(m-r_{t}\right) \pi\right) x J_{x}^{p} \\
& \left.+\frac{1}{2}\left(\frac{b_{1} \sigma_{t}+\gamma_{t}}{\sigma_{t}}\right)^{2} J_{m m}^{p}+\frac{1}{2} \sigma_{t}^{2} \pi^{2} x^{2} J_{x x}^{p}+\left(b_{1} \sigma_{t}+\gamma_{t}\right) \pi x J_{m x}^{p}\right),
\end{aligned}
$$

where $J_{t}^{p}, J_{m}^{p}, J_{x}^{p}, J_{m m}^{p}$, and so forth. represent the corresponding first- and second-order derivatives of $J^{p}$ and the upper index $p$ indicates that this is the value function under partial information. We will later also consider the value function under full information, which we will denote with $J^{f}$. If the optimal solution exists, then $J_{x x}^{p}<0$ and the maximum on the right-hand side of (7.2) is attained at

$$
\pi_{p}^{*}=-\frac{\left(m-r_{t}\right) J_{x}^{p}+\left(b_{1} \sigma_{t}+\gamma_{t}\right) J_{m x}^{p}}{\sigma_{t}^{2} x J_{x x}^{p}}
$$

Substituting (7.3) back into the HJB equation (7.2), we find that the value function under partial information satisfies the following partial differential equation:

$$
J_{t}^{p}+a m J_{m}^{p}+r_{t} x J_{x}^{p}+\frac{1}{2}\left(\frac{b_{1} \sigma_{t}+\gamma_{t}}{\sigma_{t}}\right)^{2} J_{m m}^{p}-\frac{\left(\left(m-r_{t}\right) J_{x}^{p}+\left(b_{1} \sigma_{t}+\gamma_{t}\right) J_{m x}^{p}\right)^{2}}{2 \sigma_{t}^{2} J_{x x}^{p}}=0
$$

with the terminal condition:

$$
J^{p}(T, m, x)=\frac{x^{\beta}}{\beta}
$$

In the following we will show how to reduce the PDE (7.4) to a system of three ODEs and in this way obtain a semianalytic form for the value function under partial information. In order to do this, let us first consider the reduction

$$
J^{p}(t, m, x)=H(t, m) \cdot \frac{x^{\beta}}{\beta}
$$

Substitution into (7.4) gives

$$
\begin{aligned}
& H_{t} \frac{x^{\beta}}{\beta}+ a m H_{m} \frac{x^{\beta}}{\beta}+r x^{\beta} H+\frac{1}{2}\left(\frac{b_{1}+\gamma_{t}}{\sigma}\right)^{2} H_{m m} \frac{x^{\beta}}{\beta} \\
&-\frac{\left((m-r) H x^{\beta-1}+\left(b_{1} \sigma+\beta_{t}\right) H_{m} x^{\beta-1}\right)^{2}}{2 \sigma^{2} H(\beta-1) x^{\beta-2}}=0 .
\end{aligned}
$$


We see that the common factor $x^{\beta} / \beta$ cancels out and that (7.7) can be rewritten as

$$
\frac{x^{\beta}}{\beta}\left\{H_{t}+a m H_{m}+r \beta H+\frac{1}{2}\left(\frac{b_{1}+\gamma_{t}}{\sigma}\right)^{2} H_{m m} \frac{x^{\beta}}{\beta}-\frac{\left(\beta(m-r) H+\left(b_{1} \sigma+\gamma_{t}\right) H_{m}\right)^{2}}{2 \sigma^{2} H(\beta-1)}\right\}=0 .
$$

Using the notation $\alpha(t)$ for the deterministic function

$$
\alpha(t):=\frac{b_{1} \sigma+\gamma_{t}}{\sigma}
$$

as well as discounting the function $H(t, m)$ with an appropriate discount factor, that is,

$$
\widetilde{H}(t, m):=e^{r \beta t} H(t, m),
$$

we obtain from (7.8), (7.6), and (7.5) that

$$
\begin{gathered}
\widetilde{H}_{t}+a m \widetilde{H}_{m}+\frac{1}{2} \alpha(t)^{2} \widetilde{H}_{m m}-\beta \frac{\left[((m-r) / \sigma) \widetilde{H}+\alpha(t) \widetilde{H}_{m}\right]^{2}}{2(\beta-1) \widetilde{H}}=0, \\
\widetilde{H}(T, m)=e^{r \beta T} .
\end{gathered}
$$

Looking at the PDE (7.11) with boundary condition (7.12) we make the following sophisticated guess:

$$
\widetilde{H}(t, m)=e^{A_{p}(t) m^{2}+B_{p}(t) m+C_{p}(t)},
$$

with $A_{p}(t), B_{p}(t)$, and $C_{p}(t)$ function of $t$ only satisfying $A_{p}(T)=B_{p}(T)=0$, and $C_{p}(T)=r \beta T$. The subindex $p$ indicates that we deal with the case of partial information. We conclude that

$$
\begin{gathered}
\widetilde{H}_{t}(t, m)=\widetilde{H}(t, m)\left(A_{p}^{\prime}(t) m^{2}+B_{p}^{\prime}(t) m+C_{p}^{\prime}(t)\right), \\
\widetilde{H}_{m}(t, m)=\widetilde{H}(t, m)\left(2 A_{p}(t) m+B_{p}(t)\right), \\
\widetilde{H}_{m m}(t, m)=\widetilde{H}(t, m)\left[\left(2 A_{p}(\mathrm{t}) m+B_{p}(t)\right)^{2}+2 A(t)\right],
\end{gathered}
$$


and substitution of these expressions into (7.11) gives

$$
\begin{aligned}
\widetilde{H}(t, m) & \left(A_{p}^{\prime}(t) m^{2}+B_{p}^{\prime}(t) m+C_{p}^{\prime}(t)\right)+\left(a_{1} m+a_{0}\right) \widetilde{H}(t, m)\left(2 A_{p}(t) m+B_{p}(t)\right) \\
+ & \frac{\alpha^{2}(t)}{2} \widetilde{H}(t, m)\left[\left(2 A_{p}(t) m+B_{p}(t)\right)^{2}+2 A(t)\right] \\
& -\frac{\beta\left[((m-r) / \sigma) \widetilde{H}(t, m)+\alpha(t) \widetilde{H}(t, m)\left(2 A_{p}(t) m+B_{p}(t)\right)\right]^{2}}{2(\beta-1) \widetilde{H}(t, m)}=0 .
\end{aligned}
$$

Rearranging terms according to the order of $m$ gives

$$
\begin{aligned}
& {\left[A_{p}^{\prime}(t)+2 a_{1} A_{p}(t)+2 \alpha^{2}(t) A_{p}^{2}(t)-\frac{\beta\left(2 \alpha(t) A_{p}(t)+1 / \sigma\right)}{2(\beta-1)}\right] m^{2}} \\
& +\left[B_{p}^{\prime}(t)+a_{1} B_{p}(t)+2 \alpha^{2}(t) A_{p}(t) B_{p}(t)+2 a_{0} A_{p}(t)\right. \\
& \left.\quad-\frac{\beta\left(2 \alpha(t) A_{p}(t)+1 / \sigma\right)\left(\alpha(t) B_{p}(t)-r / \sigma\right)}{(\beta-1)}\right] m \\
& +\left[C_{p}^{\prime}(t)+\frac{\alpha^{2}(t)}{2}\left(2 A_{p}(t)+B_{p}^{\prime}(t)\right)+a_{0} B_{p}(t)-\frac{\beta\left(\alpha(t) B_{p}(t)-r / \sigma\right)^{2}}{2(\beta-1)}\right]=0 .
\end{aligned}
$$

The latter can only be identical zero, if the three brackets are identical zero. This provides us with three first-order ODEs for the functions $A_{p}(t), B_{p}(t)$ and $C_{p}(t)$ :

$$
\begin{gathered}
A_{p}^{\prime}(t)=\frac{\beta}{2(\beta-1)}\left(2 \alpha(t) A_{p}(t)+\frac{1}{\sigma}\right)^{2}-2\left(a_{1}+\alpha^{2}(t) A_{p}(t)\right) A_{p}(t), \\
B_{p}^{\prime}(t)=\frac{\beta}{\beta-1}\left(2 \alpha(t) A_{p}(t)+\frac{1}{\sigma}\right)\left(\alpha(t) B_{p}(t)-\frac{r}{\sigma}\right)-\left(a_{1}+2 \alpha^{2}(t) A_{p}(t)\right) B_{p}(t)-2 a_{0} A_{p}(t), \\
C_{p}^{\prime}(t)=\frac{\beta}{2(\beta-1)}\left(\alpha(t) B_{p}(t)-\frac{r}{\sigma}\right)^{2}-\frac{\alpha^{2}(t)}{2}\left(2 A_{p}(t)+B_{p}^{2}(t)\right)-a_{0} B_{p}(t) .
\end{gathered}
$$

The equation for $A_{p}(t)$ is a Riccati ODE, however due to the presence of the function $\alpha(t)$ it cannot be solved explicitly. The ODEs for $B_{p}(t)$ and $C_{p}(t)$ are linear. It would be possible to write down an analytic expression for their solutions, however they would depend on an integral of $A_{p}(t)$, which one would still need to compute numerically. The errors due to the discretization of the integral and the numerical error in $A_{p}(t)$ when computing this integral are of about the same order as when applying a standard Runge-Kutta scheme right from the beginning, which is what we did in our numerical analysis. 
Let us now move to the case of full information. In this case the agent maximizes $\mathbb{E}\left\{x^{\beta}(T) / \beta\right\}$ under the constraint (2.6). We denote the value function of the corresponding stochastic optimal control problem with

$$
J^{f}(t, \mu, x)=\max _{\left\{\pi_{u} \in \mathcal{F}_{u}\right\}_{t \leq u \leq T}} \mathbb{E}\left[U\left(X_{T}\right) \mid, \mu_{t}=\mu, X_{t}=x\right] .
$$

Note that for the case $t=0$ this is the value function of the informed agent, once he knows the realization of $\mu_{0}$, which by Lemma 3.2. is normal distributed. We pick up on this point later.

The HJB equation for this problem is given by

$$
0=\max _{\pi}\left\{J_{t}^{f}+\frac{\sigma^{2} x^{2} \pi^{2}}{2} J_{x x}^{f}+[r+(\mu-r) \pi] x J_{x}^{f}+\left(a_{1} \mu+a_{0}\right) J_{\mu}^{f}+\frac{b_{1}^{2}+b_{2}^{2}}{2} J_{\mu \mu}^{f}+b_{1} \sigma \pi x J_{\mu x}^{f}\right\} .
$$

Assuming that $J_{x x}^{f}$ is negative, the maximizer is given by

$$
\pi_{f}^{*}=-\frac{(\mu-r) J_{x}^{f}(t, \mu, x)+b_{1} \sigma J_{\mu x}^{f}(t, \mu, x)}{\sigma^{2} x J_{x x}^{f}(t, \mu, x)} .
$$

Note that as one would expect, the maximizer formally coincides with the maximizer under partial information (7.3) in the case that $\gamma(t)=0$, replacing $m(t)$ by $\mu(t)$. Substituting $\pi_{f}^{*}$ in (7.19) implies

$$
J_{t}^{f}+r x J_{x}^{f}+\left(a_{1} \mu+a_{0}\right) J_{\mu}^{f}+\frac{b_{1}^{2}+b_{2}^{2}}{2} J_{\mu \mu}^{f}-\frac{\left[(\mu-r) J_{x}^{f}+b_{1} \sigma J_{\mu x}^{f}\right]^{2}}{2 \sigma^{2} J_{x x}^{f}}=0
$$

with terminal condition

$$
J^{f}(T, \mu, x)=\frac{x^{\beta}}{\beta} .
$$

Inspired by the analysis of the case $J^{p}(t, m, x)$, we make the sophisticated guess:

$$
J^{f}(t, \mu, x)=e^{A_{f}(t) \mu^{2}+B_{f}(t) \mu+C_{f}(t)} \frac{x^{\beta}}{\beta}
$$


Substitution into (7.22) leads in analogy to the analysis before to the following system of three ODEs:

$$
\begin{gathered}
A_{f}^{\prime}(t)=-2 a_{1} A_{f}(t)-4 \tilde{b} A_{f}^{2}(t)+\frac{\beta}{2 \sigma^{2}(\beta-1)}\left(2 b_{1} \sigma A_{f}(t)+1\right)^{2}, \\
B_{f}^{\prime}(t)=-a_{1} B_{f}(t)-2 a_{0} A_{f}(t)-4 \tilde{b} A_{f}(t) B_{f}(t)+\frac{\beta}{\sigma^{2}(\beta-1)}\left(2 b_{1} \sigma A_{f}(t)+1\right)\left(b_{1} \sigma B_{f}(t)-r\right), \\
C_{f}^{\prime}(t)=-r \beta-a_{0} B_{f}(t)-\tilde{b}\left(2 A_{f}(t)+B_{f}^{2}(t)\right)+\frac{\beta}{2 \sigma^{2}(\beta-1)}\left(b_{1} \sigma B_{f}(t)-r\right)^{2},
\end{gathered}
$$

where $\tilde{b}=\left(b_{1}^{2}+b_{2}^{2}\right) / 2$ and $A_{f}(T)=B_{f}(T)=C_{f}(T)=0$. In summary, the maximizers for the case of full and partial information are therefore given by

$$
\begin{gathered}
\pi_{f}^{*}(t, \mu)=-\frac{\mu-r+b_{1} \sigma^{2}\left(2 A_{f}(t) \mu+B_{f}(t)\right)}{\sigma^{2}(\beta-1)}, \\
\pi_{p}^{*}(t, m)=-\frac{m-r+\left(b_{1} \sigma+\gamma(t)\right)\left(2 A_{p}(t) m+B_{p}(t)\right)}{\sigma^{2}(\beta-1)},
\end{gathered}
$$

and the corresponding value functions are given by

$$
\begin{gathered}
J^{f}(t, \mu, x)=e^{A_{f}(t) \mu^{2}+B_{f}(t) \mu+C_{f}(t)}\left(\frac{x^{\beta}}{\beta}\right), \\
J^{p}(t, m, x)=e^{A_{p}(t) m^{2}+B_{p}(t) m+C_{p}(t)-r \beta t}\left(\frac{x^{\beta}}{\beta}\right) .
\end{gathered}
$$

We can clearly see from (7.25) how the newly derived portfolio rules adjust the Merton [16] rule for the stochastic drift term under full and partial information. Note that like in the classical Merton problem, the optimal investment strategies do not depend on the level of wealth $x$, which is why this variable has not been included in the notation in (7.25).

In order to determine the value of information we now need to compute the difference between the two value functions of full information (7.26) and partial information (7.27). In order to do this, we need to realize one thing. While the initial condition $m_{0}$ for the partial information case is deterministic, the initial condition $\mu_{0}$ for the full information case is by assumption a normal distributed random variable, whose realization the fully informed agent observes, and after that behaves optimally conditional on $\mu_{0}$. According to Lemma 3.2, we know that $\mu_{0} \sim \mathcal{N}\left(m_{0}, \gamma_{0}\right)$. In average the informed agents optimal utility is therefore given by $\mathbb{E}\left\{J^{f}\left(0, \mu_{0}, x\right)\right\}$, where the expectation is taken over $\mu_{0}$. Knowing the semiexplicit form of 


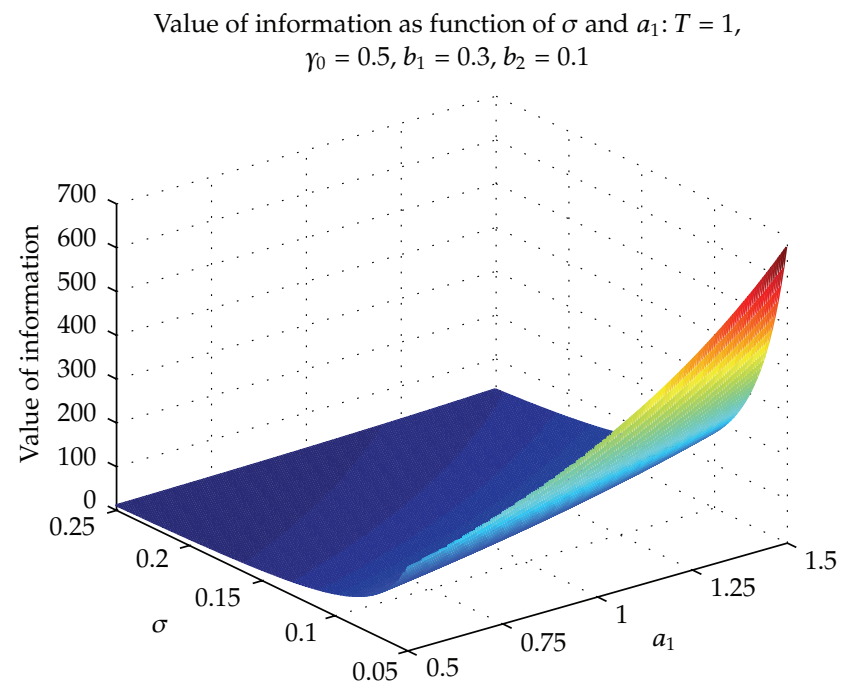

Figure 1: $\mho$ as a function of $\sigma$ and $a_{1}$.

the value function $J^{f}$ in (7.26) as well as the density function of the normal distribution, it is not to difficult to carry out this integration. The result can be computed as follows:

$$
\begin{aligned}
& \mathbb{E}\left\{e^{A_{f}(0) \mu^{2}+B_{f}(0) \mu+C_{f}(0)}\right\} \\
& \quad=\int_{-\infty}^{\infty} \frac{1}{\sqrt{2 \pi \gamma_{0}}} e^{A_{f}(0) x^{2}+B_{f}(0) x+C_{f}(0)} e^{-\left(\left(x-m_{0}\right)^{2} / 2 \gamma_{0}\right)} d x \\
&=\frac{1}{\sqrt{1-2 A_{f}(0) \gamma_{0}}} e^{\left(4 A_{f}(0) C_{f}(0) \gamma_{0}-2 C_{f}(0)-2 A_{f}(0) m_{0}^{2}-B_{f}^{2}(0) \gamma_{0}-2 B_{f}(0) m_{0}\right) / 2\left(2 A_{f}(0) \gamma_{0}-1\right)},
\end{aligned}
$$

if $1>2 A_{f}(0) \gamma_{0}$ and $\infty$ otherwise. In this way, the two value functions are now comparable and we obtain the value of information for the CRRA case:

$$
\mho_{\beta}=\mathbb{E}\left\{J^{f}\left(0, \mu_{0}, x\right)\right\}-J^{p}\left(t, m_{0}, x\right) .
$$

Note that the value of information is infinite, if $1 \leq 2 A_{f}(0) \gamma_{0}$. The latter however could not be observed for realistic parameters.

In the numerical examples presented in the appendix we assume that $r=0.08, a_{1} \in$ $[-1,1], a_{0}=0.1, b_{1} \in[-0.5,1.2], b_{2} \in[-0.3,0.3], \beta \in[0.1,0.4], \sigma \in[0.2,0.4], \gamma_{0} \in[0,0.2]$, $T=0.25, m_{0}=0.2$ and $x=0.4$. We observe that the value of information is increasing in the volatility parameter $\sigma$ as well as in the risk aversion parameter $\beta$. This means that more risk averse agents value information higher than less risk averse agents, which makes sense from an intuitive point of view. The value of information is further increasing in $\gamma_{0}$ which is the initial uncertainty in $\mu_{0}$. 


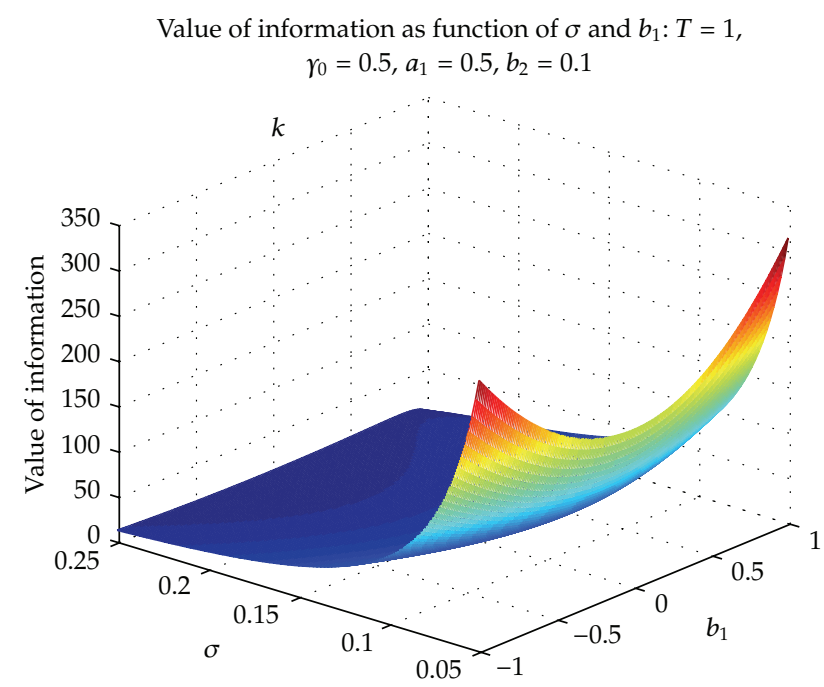

Figure 2: $\mho$ as a function of $\sigma$ and $b_{1}$.

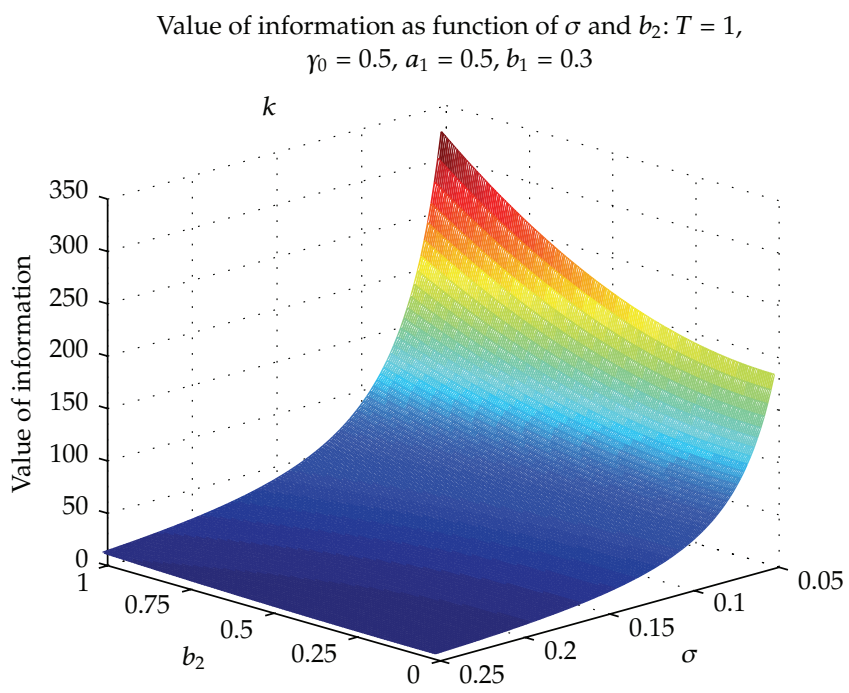

Figure 3: $\mho$ as a function of $\sigma$ and $b_{2}$.

\section{Conclusions}

We have studied the value of full information in a financial market model with partial information, where the drift rate of the risky asset is assumed to be an unobservable dynamic process, which we model as the solution of a linear stochastic differential equation with constant coefficients. We derived an analytic formula for the value of information taking logarithmic utility from terminal wealth as an objective, and a semianalytical formula for the case of CRRA-utility from terminal wealth. We performed a detailed comparative statics and singled out the various effects the model parameters have on the value of information. 


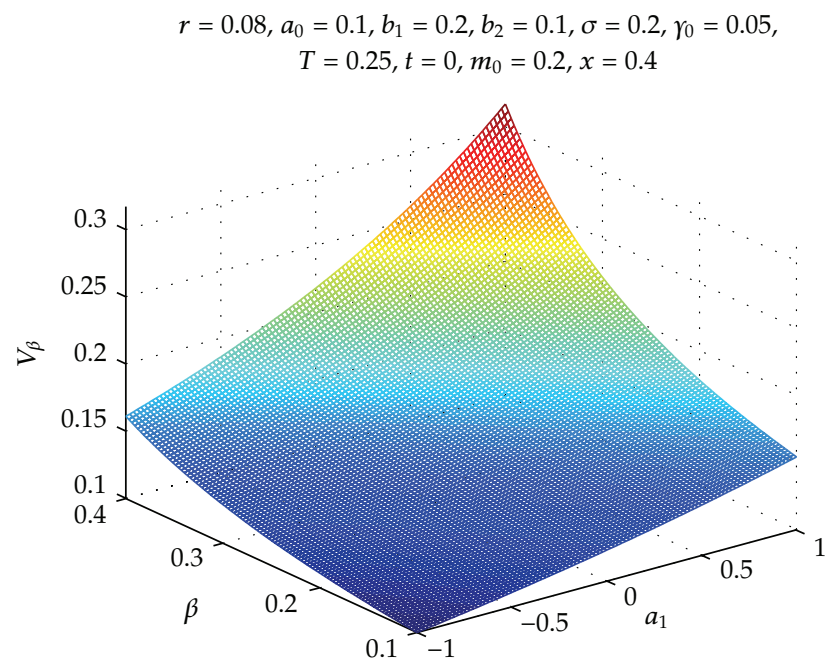

Figure 4: $\mho_{\beta}$ as a function of $a_{1}$ and $\beta$.

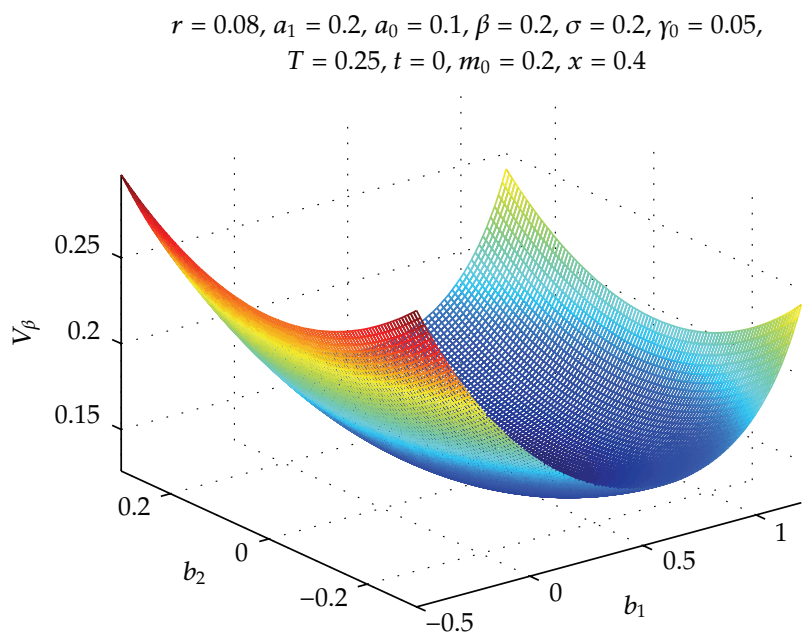

Figure 5: $\mho_{\beta}$ as a function of $b_{1}$ and $b_{2}$.

\section{Appendices}

\section{A. Logarithmic Utility}

(See Figures 1, 2, and 3.)

\section{B. CRRA Utility}

(See Figures 4, 5, and 6.) 


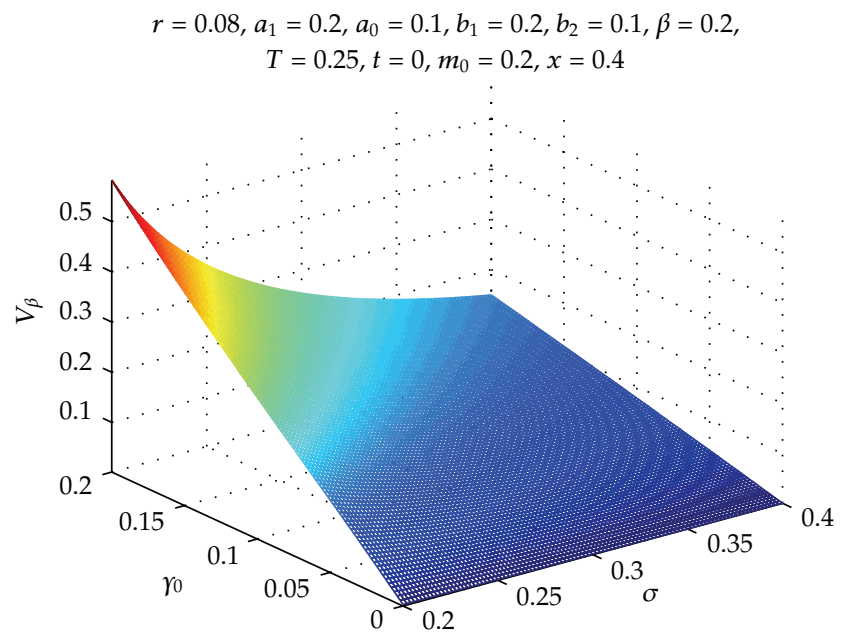

Figure 6: $\mho_{\beta}$ as a function of $\sigma$ and $\gamma_{0}$.

\section{Acknowledgments}

This research is supported by the national Natural Science Foundation of China (70971037) and the Rheinland Pfalz Excellence cluster DASMOD. Z. Yang would like to thank Professor Jie Xiong from the University of Tennessee for many helpful comments. All authors would like to thank two anonymous referees for their valuable feedback and suggestions.

\section{References}

[1] U. Birchler and M. Büttler, Information Economics, Routledge, Taylor \& Francis, London, UK, 2007.

[2] J. Hirshleifer and J. G. Riley, The Analytics of Uncertainty and Information, CUP, Cambridge, UK, 1992.

[3] J. Amendinger, D. Becherer, and M. Schweizer, "A monetary value for initial information in portfolio optimization," Finance and Stochastics, vol. 7, no. 1, pp. 29-46, 2003.

[4] P. Imkeller, "Malliavin's calculus in insider models: additional utility and free lunches," Mathematical Finance, vol. 13, no. 1, pp. 153-169, 2003.

[5] C.-O. Ewald, "Optimal logarithmic utility and optimal portfolios for an insider in a stochastic volatility market," International Journal of Theoretical and Applied Finance, vol. 8, no. 3, pp. 301-319, 2005.

[6] A. Kohatsu-Higa and A. Sulem, "Utility maximization in an insider influenced market," Mathematical Finance, vol. 16, no. 1, pp. 153-179, 2006.

[7] J. Jacod, "Groissement initial, hypothese $\left(\mathrm{H}^{\prime}\right)$ et theoreme de Girsanov," in Groissements des Filtrations: Exemples et Applications, T. Jeulin and M. Yor, Eds., vol. 1117 of Lecture Notes in Mathematics, pp. 298409, Springer, Berlin, Germany, 1985.

[8] C. O. Ewald and Y. Xiao, "Information: price and impact on general welfare and optimal investment. An anticipative stochastic differential game model," preprint, 2010, http:/ / papers.ssrn.com/sol3/papers.cfm?abstract_id=985047.

[9] H. Pham and M.-C. Quenez, "Optimal portfolio in partially observed stochastic volatility models," The Annals of Applied Probability, vol. 11, no. 1, pp. 210-238, 2001.

[10] J. Sass and U. G. Haussmann, "Optimizing the terminal wealth under partial information: the drift process as a continuous time Markov chain," Finance and Stochastics, vol. 8, no. 4, pp. 553-577, 2004.

[11] G. Genotte, "Optimal portfolio choice under incomplete information," The Journal of Finance, vol. 41, no. 3, pp. 733-746, 1986.

[12] M.-U. Dothan and D. Feldman, "Equilibrium interest rates and multiperiod bonds in a partially observable economy," The Journal of Finance, vol. 41, no. 2, pp. 369-382, 1986. 
[13] J.-B. Detemple, "Asset pricing in a production economy with incomplete information," The Journal of Finance, vol. 41, no. 2, pp. 383-392, 1986.

[14] Y. Xia, "Learning about predictability: the effects of parameter uncertainty on dynamic asset allocation," Journal of Finance, vol. 56, no. 1, pp. 205-246, 2001.

[15] Z. Yang and C. Q. Ma, "Optimal trading strategy with partial information and the value of information: the simplified and generalized models," International Journal of Theoretical and Applied Finance, vol. 4, no. 5, pp. 759-772, 2001.

[16] R. C. Merton, "Lifetime portfolio selection under uncertainty: the continuous time case," The Review of Economics and Statistics, vol. 51, no. 3, pp. 247-257, 1969.

[17] R. S. Liptser and A. N. Shiryayev, Statistics of Random Processes, Springer, New York, NY, USA, 1977. 


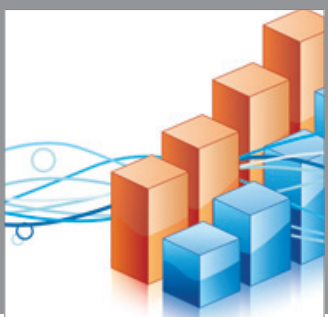

Advances in

Operations Research

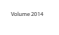

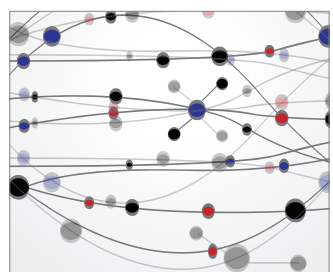

\section{The Scientific} World Journal
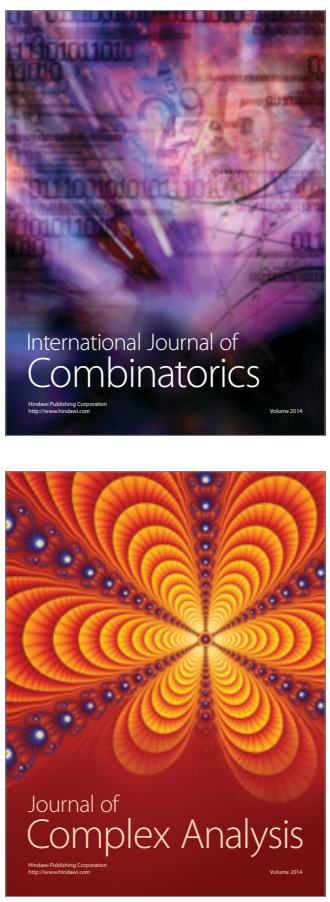

International Journal of

Mathematics and

Mathematical

Sciences
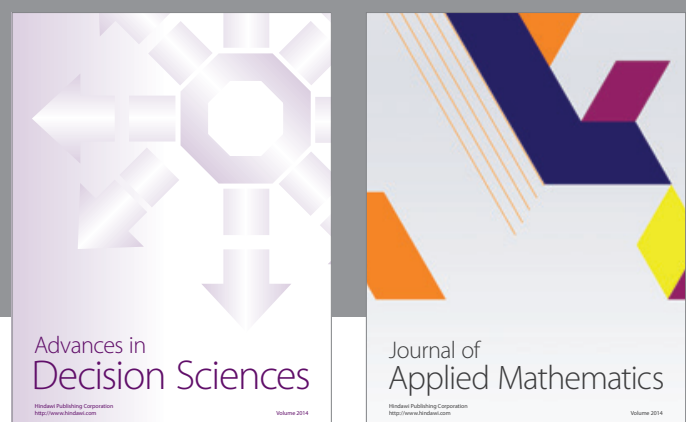

Journal of

Applied Mathematics
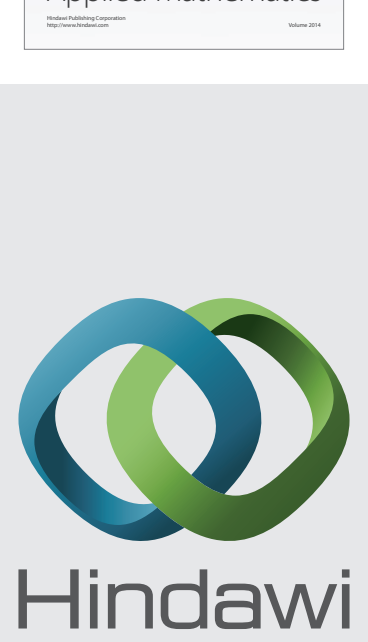

Submit your manuscripts at http://www.hindawi.com
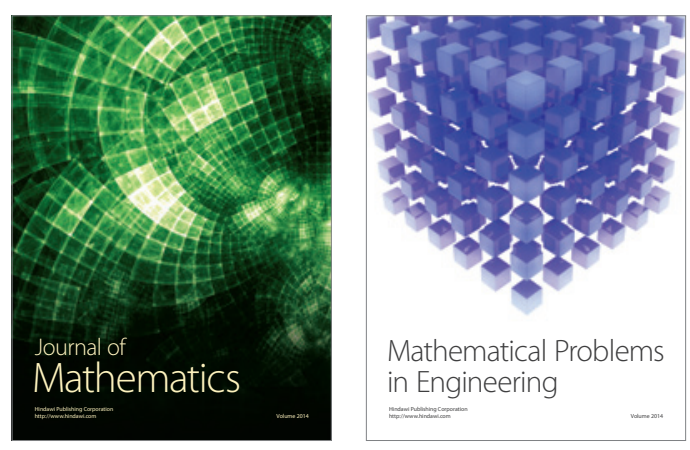

Mathematical Problems in Engineering
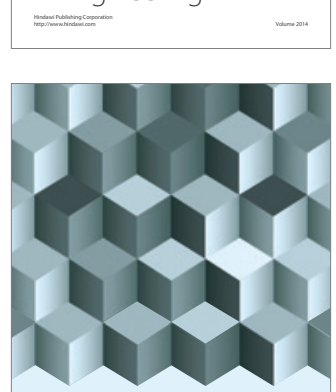

Journal of

Function Spaces
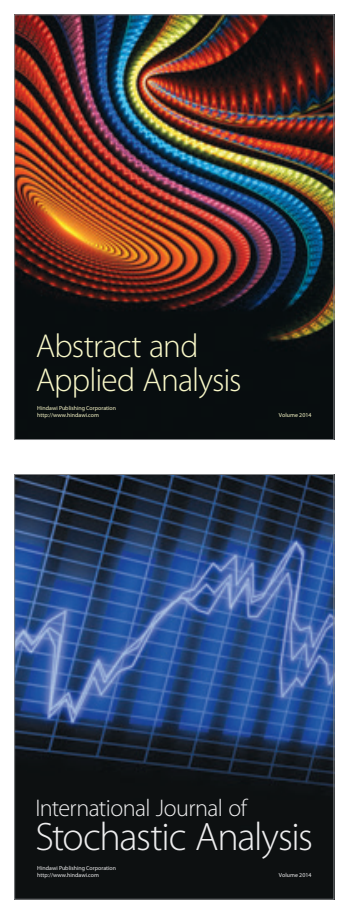

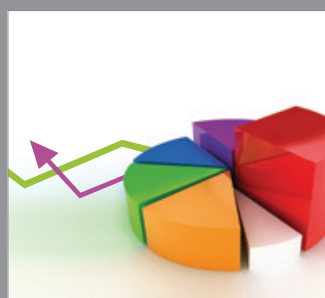

ournal of

Probability and Statistics

Promensencen
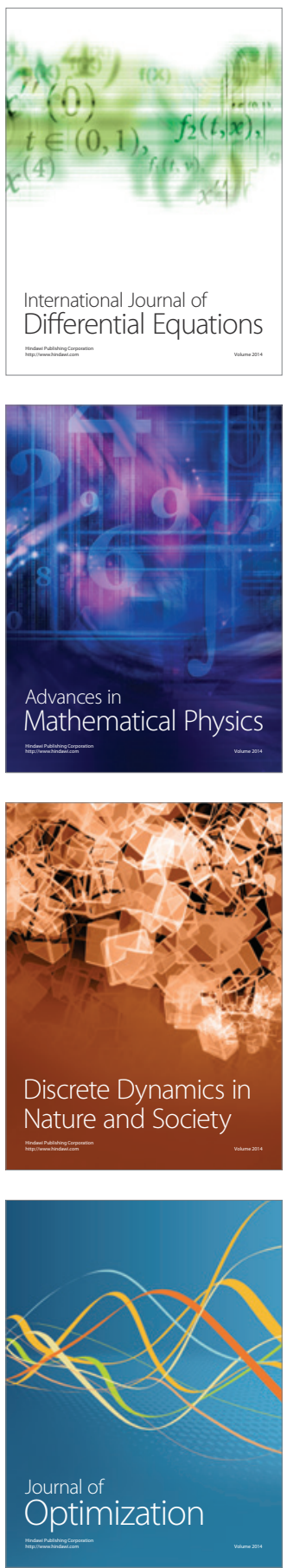